\title{
Inconspicuous longitudinal tears of the intracranial vertebral artery in traumatic basal subarachnoid hemorrhage
}

\author{
Seongho Kim \\ Central Forensic Medical Center, National Forensic Service, Wonju, Korea
}

\begin{abstract}
Blunt force trauma to the head or neck region can cause traumatic basal subarachnoid hemorrhage (TBSAH), which can result in rapid loss of consciousness and death; however, detecting such a vascular injury is difficult. Posterior neck dissection was performed to investigate the bleeding focus in TBSAH cases 2018 and 2019. In all four cases, autopsies revealed a longitudinal tear in the midsection of the vertebral artery's intracranial portion. The midportion of the intracranial vertebral artery appears to be most vulnerable to TBSAH. Interestingly, three of the cases showed only a vaguely visible longitudinal fissure in the artery without a grossly apparent tear; rupture was confirmed by microscopic examination. Longitudinal fissures of the intracranial vertebral artery, which are difficult to identify without detailed examination, may be overlooked in some cases of TBSAH. Thus, careful gross and microscopic examination of the vertebral artery is recommended in cases of TBSAH.
\end{abstract}

Key Words: Vertebral artery; Rupture; Basal subarachnoid hemorrhage; Trauma

Received: August 29, 2019 Revised: September 30, 2019 Accepted: October 15, 2019

Corresponding Author: Seongho Kim, MD, Central Forensic Medical Center, National Forensic Service, 10 Ipchun-ro, Wonju 26460, Korea

Tel: +82-42-866-4551, Fax: +82-42-866-4569, E-mail: rivebt99@gmail.com

Blunt force trauma to the head or neck region can cause traumatic basal subarachnoid hemorrhage (TBSAH). TBSAH can be fatal, as it can lead to instantaneous loss of consciousness and death. The most common site of vascular rupture is thought to be the vertebral artery, particularly the intracranial course $[1,2]$. However, because of the position of the vertebral artery in the cranial cavity, it is difficult to determine the bleeding focus on routine autopsy; also, the intracranial vertebral arteries are normally cut to remove the brain. In TBSAH cases, posterior neck dissection has been used to locate the bleeding focus in the dura-perforating and intracranial portions of the vertebral artery; using this method, tear sites in the vertebral artery can be identified more frequently [3,4]. In this report, the clinicopathologic features of four TBSAH cases are summarized.

\section{CASE REPORT}

Posterior neck dissection was performed during autopsy in four cases with a history of TBSAH by the National Forensic Service in South Korea from 2018 to 2019 to investigate bleeding focus.
The deceased individuals were two men and two women aged 22-53 years. Another case of basal subarachnoid hemorrhage with a trauma history was excluded from this series of four cases because of underlying vasculopathy (aneurysmal rupture).

The posterior neck dissection method was previously described. Briefly, the posterior laminae of the upper cervical vertebrae are excised; then, an inverted wedge is cut from the posterior occipital bone above the foramen magnum. After the dura are opened and the spinal cord is exposed, the spinal cord is pulled to one side and the dura surrounding the penetrating portion of the vertebral artery is excised. Then, the intracranial vertebral artery is pulled up and the extracranial vertebral artery is severed just below the dura. The brain, spinal cord, and dissected intra- and extracranial vertebral arteries are removed together [3]. Finally, the dissected vessels are carefully examined to identify the source of hemorrhage.

The deceased individuals rapidly lost consciousness after being assaulted on the face, head, or neck. All of the deceased individuals were under the influence of alcohol. Two individuals died within 24 hours, whereas the other two individuals survived for two to 
eight days on life support including mechanical ventilation. Autopsy revealed diffuse basal subarachnoid hemorrhage in all four cases, with vertebral artery injury. In all four cases of vertebral artery injury, a longitudinal tear was identified in the midportion of the vessels' intracranial portion. Interestingly, three of the four intracranial vertebral artery injury cases had a longitudinal fissure in the vertebral artery instead of a grossly evident tear; rupture was confirmed by the water injection method or microscopic examination. Neither postmortem computed tomography (CT) nor postmortem angiography was performed in any of the cases. The clinicopathologic findings are summarized in Table 1.

\section{Case 1}

A 58-year-old male argued with another male, was punched in the head and immediately lost consciousness. Cardiopulmonary resuscitation was performed in the hospital but he died approximately 1 hour after the assault. The postmortem examination showed muscular hemorrhage in the left infraauricular area and diffuse basal subarachnoid hemorrhage. The intracranial arteries in the basal portion of the brain were carefully examined. A vaguely visible longitudinal fissure was found in the midportion of the left intracranial vertebral artery. After injecting water into the proximal portion of the vertebral artery with a syringe, water leakage was noted along the longitudinal fissure. Fibrinous

Table 1. Clinicopathologic findings of 4 TBSAH cases

\begin{tabular}{|c|c|c|c|c|c|c|c|c|c|}
\hline Case No. & $\begin{array}{c}\text { Sex/ } \\
\text { age (yr) }\end{array}$ & Main site of impact & Relevant autopsy findings & $\begin{array}{l}\text { Cause of } \\
\text { impact }\end{array}$ & Survival & $\begin{array}{l}\text { Injured } \\
\text { vessel }\end{array}$ & $\begin{array}{c}\text { Portion } \\
\text { of the injured } \\
\text { vessel }\end{array}$ & Shape of injury & Drinking \\
\hline 1 & $\mathrm{M} / 53$ & Lt infra-auricular & $\begin{array}{l}\text { Intramuscular hemorrhage in the left } \\
\text { infra-auricular lesion }\end{array}$ & Fist & $1 \mathrm{hr}$ & Lt IVA & Mid 1/3 & $\begin{array}{l}\text { Longitudinal } \\
\text { fissure } 7 \mathrm{~mm}\end{array}$ & + \\
\hline 2 & $\mathrm{~F} / 30$ & Lt posterior neck & $\begin{array}{l}\text { Intramuscular hemorrhage in the left } \\
\text { posterior neck }\end{array}$ & Fist & $23 \mathrm{hr}$ & Lt IVA & Mid 1/3 & $\begin{array}{l}\text { Longitudinal } \\
\quad \text { fissure } 5 \mathrm{~mm}\end{array}$ & + \\
\hline 3 & $\mathrm{~F} / 22$ & Lt infra-auricular & $\begin{array}{l}\text { Contusions and intramuscular } \\
\text { hemorrhage in the left infra-auricular } \\
\text { lesion and mandible } \\
\text { Multiple contusions of the face, chest, } \\
\text { arm, and thigh } \\
\text { Stent and coils in the ruptured vertebral } \\
\text { artery }\end{array}$ & Fist & 2 days & Lt IVA & Mid and distal & $\begin{array}{l}\text { Longitudinal } \\
14 \mathrm{~mm}\end{array}$ & + \\
\hline 4 & $\mathrm{M} / 41$ & $\begin{array}{l}\text { Face and head, } \\
\text { multiple }\end{array}$ & $\begin{array}{l}\text { Multiple contusion, laceration, and } \\
\text { intramuscular hemorrhage involving } \\
\text { head and neck } \\
\text { Fractures of the mandible and maxilla }\end{array}$ & Fist knee & 8 days & Lt IVA & Mid 1/3 & $\begin{array}{l}\text { Longitudinal } \\
\quad \text { fissure } 5 \mathrm{~mm}\end{array}$ & + \\
\hline
\end{tabular}

TBSAH, traumatic basal subarachnoid hemorrhage; M, male; Lt, left; IVA, intracranial vertebral artery; F, female.
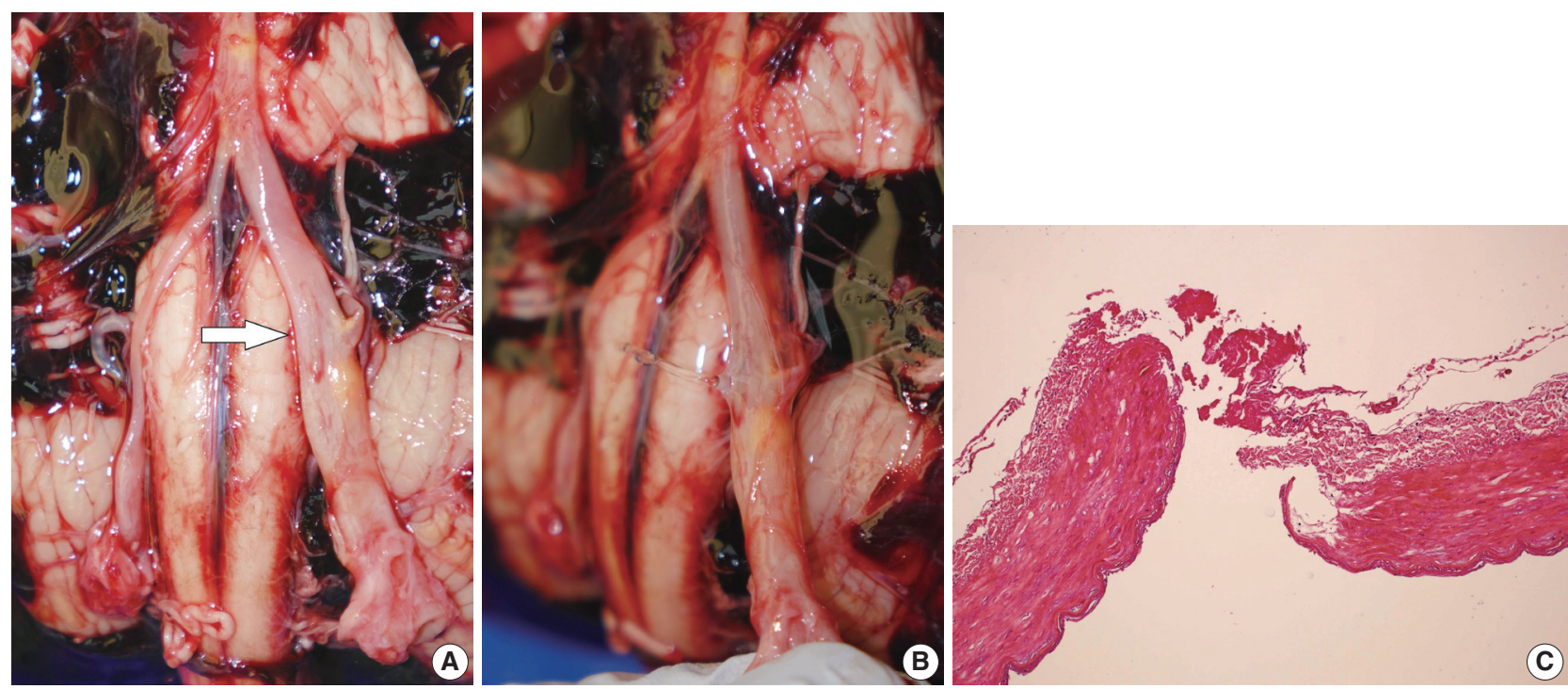

Fig. 1. (A) A vague longitudinal fissure in the left vertebral artery (white arrow). (B) Water leakage through the ruptured portion. (C) Histology of the left vertebral artery at the point of rupture showing fibrinous material at the rupture site. 
material was identified in the ruptured portion of the vessel on microscopic examination (Fig. 1).

\section{Case 2}

A 30-year old female was punched in the head. She immediately lost consciousness and was taken to the hospital. She died 23 hours after the assault. Postmortem examination revealed a deep muscular hemorrhage in the left posterior neck and diffuse basal subarachnoid hemorrhage. A vaguely visible longitudinal fissure was found in the midportion of the left intracranial vertebral artery. Water was injected into the proximal portion of the vertebral artery, but did not leak through the longitudinal fissure. However, water leakage was observed in the basilar artery. Microscopically, the longitudinal fissure of the vertebral artery was completely clogged with fibrin clots. In the area of the basilar artery where the water escaped, neither neutrophil infiltration nor fibrin materials were observed. Thus, the longitudinal fissure in the vertebral artery was identified as the true site of rupture (Fig. 2).

\section{Case 3}

A 22-year-old female was punched on the left side of the neck. She immediately lost consciousness and was taken to the hospital. CT and cerebral angiography were performed and revealed injury to the left vertebral artery. A stent and coils were inserted in the injured artery, but the patient died 2 days after the assault. Postmortem examination revealed muscular hemorrhage in the left infra-auricular area and diffuse basal subarachnoid hemorrhage. A longitudinal rupture was observed in the left vertebral artery and a stent and coils were visible in the affected artery.

\section{Case 4}

A 41-year-old man was punched on the left side of his jaw, and he collapsed. He was then struck in the face and head several times by the perpetrator's fist and knee. The victim was taken to the hospital but died eight days after the assault. Postmortem examination revealed multiple contusions of the face and head, along with fractures of the mandible and maxilla. A longitudinal fissure was found in the midportion of the left intracranial vertebral artery. Water was injected into the proximal portion of the vertebral artery, but did not leak through the longitudinal fissure. Microscopically, fibrinous material was identified in the ruptured portion of the vessel, and the lumen of the injured vessel was clogged with thrombus (Fig. 3).

Decedents are generally not considered to be human subjects for the purposes of research, and therefore are not generally under the purview of an institutional review board. For these autopsy case reports, consent was obtained for autopsy, and further informed consent was not required.

\section{DISCUSSION}

Previously, extracranial vertebral artery rupture was thought to cause fatal traumatic subarachnoid hemorrhage [5,6]. However, Leadbeatter suggested that extracranial vertebral artery injury could be a concomitant lesion and that the bleeding focus might be an unidentified vascular tear inside the cranial cavity [7]. Currently, many pathologists believe that the more common site of rupture in TBSAH is the intracranial vertebral artery $[2,8]$.
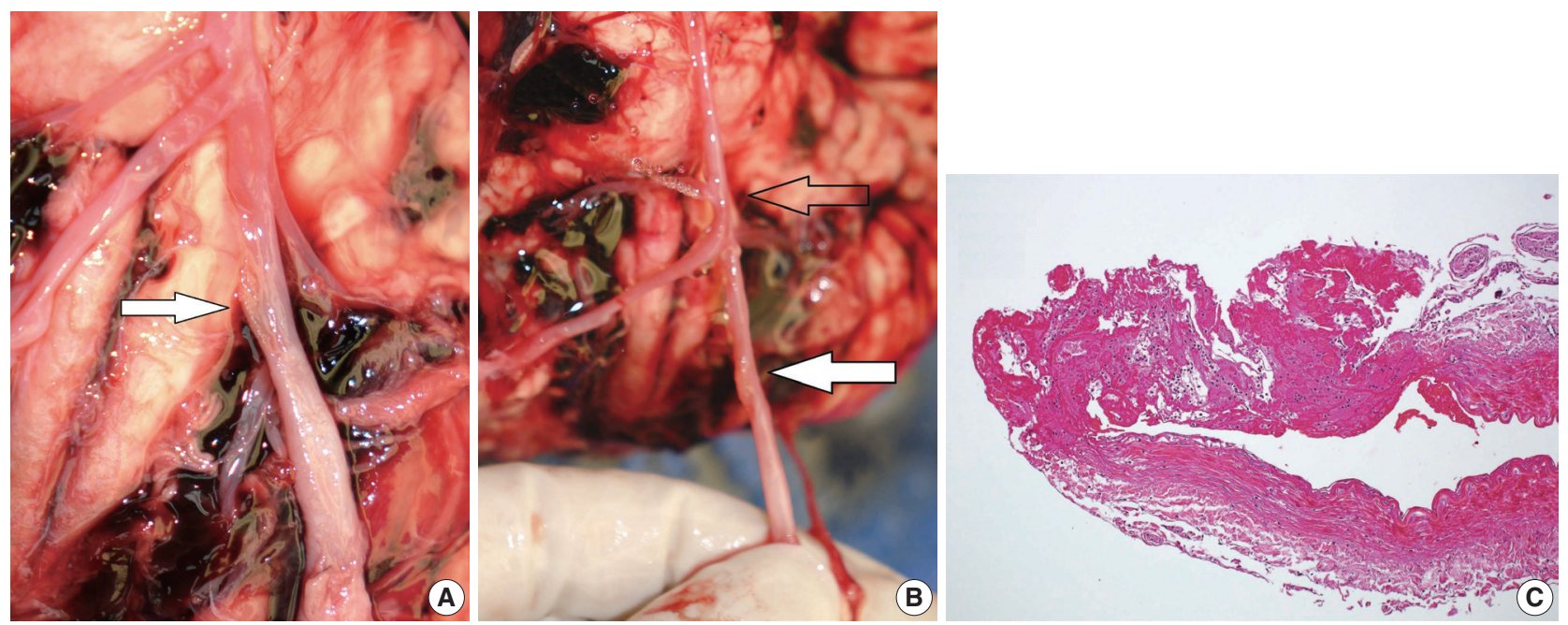

Fig. 2. (A) Longitudinal fissure in the left vertebral artery (white arrow). (B) Water leakage through the artificially ruptured portion of the proximal basilar artery (empty arrow). The true site of rupture is indicated (white arrow). (C) Histology of the true rupture site showing complete blockage by a fibrinous plug. 

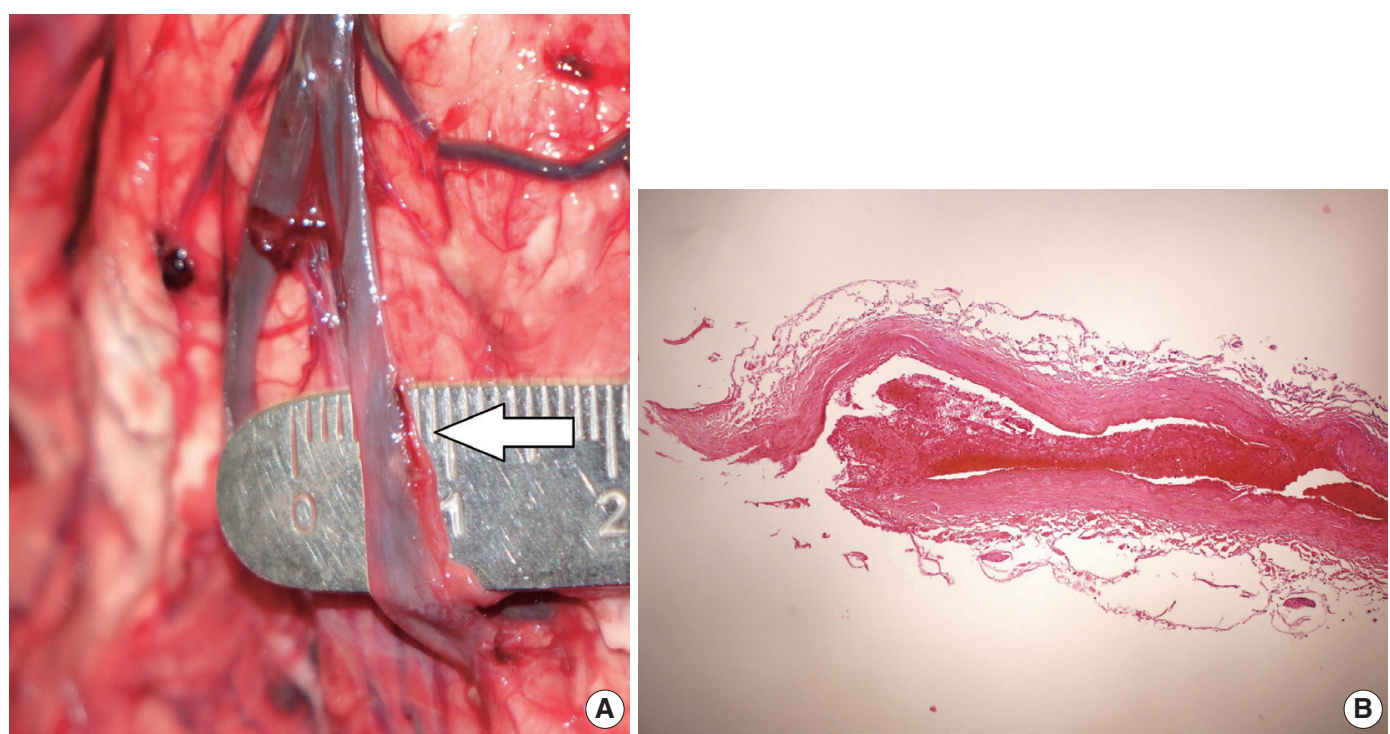

Fig. 3. (A) Longitudinal fissure in the left vertebral artery (white arrow). (B) Histology of the ruptured portion showing thrombus in the vascular lumen.

Because of the deep location of the vertebral artery in the cranial cavity, it is difficult to examine on autopsy. In case of vertebral artery injury, it can be challenging to locate the bleeding focus. When the injured vertebral artery portion is not visible on autopsy, a water injection method is commonly used, which identifies the rupture site via water leakages after injection into the origin of the vertebral artery [9].

Posterior neck dissection was used to investigate the bleeding focus in 11 TBSAH cases between 2013 and 2019, including previously published cases and the four cases described herein; intracranial vertebral artery laceration was identified in nine cases $[3,4]$. All of the deceased individuals were under the influence of alcohol, were struck in the head or neck via punching or kicking, and lost consciousness instantaneously during the assault. The individuals lived less than 24 hours in three cases and survived from 2 to 9 days in eight cases. In the past, most TBSAH patients died soon after loss of consciousness; however, advanced life support including mechanical ventilation now enables sustained survival in an unconscious state.

Longitudinal tears were identified in all nine injured intracranial vertebral arteries in this series. These findings agree with those of other studies reporting that longitudinal tears are the characteristic intracranial vertebral arterial injury in TBSAH $[10,11]$. Vascular tears in nine of the cases were observed in the midportion of the intracranial vertebral artery; in one of these cases, the tear was seen in the mid and distal portions of the intracranial course, and in another in the mid and proximal portions [4]. Although the mechanism remains unclear, based on these results, the midportion of the intracranial vertebral artery seems to be the portion most vulnerable to TBSAH. Therefore, when autopsy is performed in cases of TBSAH, it is necessary to pay close attention to the midportion of the intracranial vertebral artery. In TB$\mathrm{SAH}$, the suggested mechanisms for the vertebral artery injury are direct trauma to the vessel, extensive stretching of the vessel during hyperextension or rotation of the neck, oscillation of the brain with shearing force, and increased intravascular pressure [8]. Considering that vertebral artery injury is observed mainly in the middle portion of the intracranial regions, stretching or shearing forces on the vessels are likely to play an important role.

Three of four verified intracranial vertebral artery ruptures consisted of vaguely visible longitudinal fissures in the vertebral artery without a grossly evident tear. On gross examination, the longitudinal fissures in the external surface of the vertebral arteries seemed rough and fluffy, as opposed to the typical smooth and glistening appearance. Because it was difficult to determine with gross examination alone whether a rupture had occurred, the rupture site was confirmed by injecting water into the blood vessel to identify areas of leakage or through microscopic examination. One of the cases showed water leakage at the longitudinal fissure. In one case, water leakage was observed in the basilar artery rather than at the site of the longitudinal fissure in the intracranial vertebral artery. Histological examination revealed that the longitudinal fissure in the vertebral artery was completely obstructed with a fibrin clot and that fibrin deposition and inflammatory cell infiltration were absent in the basilar artery. Therefore, the vertebral artery area was determined to be the true 
site of the rupture and water leakage in the basilar artery was attributed to an artificial rupture that occurred during autopsy. In another case, water leakage was not observed because of thrombotic occlusion of the vessel. A longitudinal fissure occurring after vertebral artery injury may be part of the hemostatic process caused by vasoconstriction, platelet plug formation, and fibrin deposition after vascular trauma [12]. Because this subtle injury may be difficult to identify on gross examination, longitudinal fissure may be an overlooked sign of vascular trauma in some cases of TBSAH. Therefore, sufficient attention and time should be invested in identifying minute vascular injuries during autopsy in TBSAH cases.

Generally, damage to the vertebral artery ipsilateral to the blunt impact is more frequent than damage to the vertebral artery contralateral [8]. One case in this series showed multiple contusions or muscular hemorrhages on both sides of the face, head, and neck. As a result, it was difficult to determine whether the intracranial artery injury occurred ipsilaterally or contralaterally to the site of impact. In the remaining three cases with the impact on the left side of the head or neck demonstrated injury to the left vertebral artery, showing a relationship between the site of impact and the site of vascular injury

This study has several limitations. First, the number of cases was small. Second, in two cases, the survival period was longer than that reported in previous publications, and interpretation of some of the gross and histological findings may have been affected by changes occurring in the vascular lesions during the survival period. Therefore, further study is needed to determine whether these distinctive vascular injuries are commonly observed in TBSAH cases.

In conclusion, the majority of TBSAH cases showed intracranial vertebral artery injuries; therefore, the intracranial vertebral artery should be kept intact as far as possible up to its proximal portion during autopsy. Posterior neck dissection can be performed to examine the entire intracranial vertebral artery without inflicting artificial injury. If posterior neck dissection is not performed, removing the cerebral hemisphere before cutting the vertebral artery enables examination of longer portions of the intracranial vertebral artery. After brain removal, detailed examination is needed to identify minute vascular injury. Although the mechanism remains unclear, the midportion of the intracranial vertebral artery seems to be the portion most vulnerable to TBSAH. Therefore, it is necessary to pay close attention to the midportion of the intracranial vertebral artery. If any lesions suspicious for vascular damage are found, rupture should be confirmed using the water injection method or microscopic examination.

\section{ORCID}

Seongho Kim: https://orcid.org/0000-0002-1412-7630

\section{Conflicts of Interest}

The authors declare that they have no potential conflicts of interest.

\section{Funding}

No funding to declare.

\section{REFERENCES}

1. Lee CK, Gray L, Maguire J. Traumatic vertebral artery injury: detailed clinicopathologic and morphometric analysis of 6 cases. Am J Forensic Med Pathol 2009; 30: 134-6.

2. Wong B, Ong BB, Milne N. The source of haemorrhage in traumatic basal subarachnoid haemorrhage. J Forensic Leg Med 2015; 29: 1823.

3. Kim S, Kim M, Lee BW, Kim YH, Choi YS, Seo JS. Investigation of bleeding focus in the intracranial vertebral artery with the use of posterior neck dissection method in traumatic basal subarachnoid hemorrhage. J Forensic Leg Med 2015; 34: 151-4.

4. Kim S, Jun YJ, Na JI, Kim M. Rupture portions in the vertebral artery in traumatic basal subarachnoid hemorrhage. J Forensic Leg Med 2017; 52: 221-2.

5. Contostavlos DL. Massive subarachnoid hemorrhage due to laceration of the vertebral artery associated with fracture of the transverse process of the atlas. J Forensic Sci 1971; 16: 40-56.

6. Harland WA, Pitts JF, Watson AA. Subarachnoid haemorrhage due to upper cervical trauma. J Clin Pathol 1983; 36: 1335-41.

7. Leadbeatter S. Extracranial vertebral artery injury: evolution of a pathological illusion? Forensic Sci Int 1994; 67: 33-40.

8. Ong BB, Milne N. Vertebral artery trauma. In: Rutty GN, ed. Essentials of autopsy practice: reviews, updates, and advances. Cham: Springer, 2017; 23-41.

9. McCarthy JH, Sunter JP, Cooper PN. A method for demonstrating the source of bleeding in cases of traumatic subarachnoid haemorrhage. J Pathol 1999; 187: 30A.

10. Coast GC, Gee DJ. Traumatic subarachnoid haemorrhage: an alternative source. J Clin Pathol 1984; 37: 1245-8.

11. Hiraiwa K, Sato T, Sasaki T, Mizusawa I, Nata M, Kodama N. Medico-legal aspects of traumatic injury of the vertebrobasilar artery. Neurol Med Chir (Tokyo) 2005; 45: 549-55.

12. Kumar V, Abbas AK, Aster JC. Robbins basic pathology. Philadelphia, PA: Elsevier, 2018; 101-11. 